\title{
Application in composite machine using RBF neural network based on PID control
}

\author{
Jia Chunying ${ }^{1}$, Chen Yuchen ${ }^{1}$, Ding Zhigang ${ }^{2}$ \\ ${ }^{1}$ College of Electronic and Electric Engineering, Shanghai University of Engineering Science, Shanghai, China \\ ${ }^{2}$ Shanghai Computer Software Technology Development Center, Shanghai, China
}

\section{Email address \\ Chuningiia@163.com (Jia Chunying)}

To cite this article:

Jia Chunying, Chen Yuchen, Ding Zhigang. Application in Composite Machine Using RBF Neural Network Based on PID Control. Automation, Control and Intelligent Systems. Vol. 2, No. 6, 2014, pp. 100-104. doi: 10.11648/j.acis.20140206.11

\begin{abstract}
In the absence of solvent composite machine, because the radius of drum winding and rewinding roller in the transmission process is changing. With the coiled material rolls diameter more and more large, and put the curly size getting smaller and smaller, this has the certain difficulty for the tension control. Therefore, good tension control is non solvent composite is very important. Analyzed the reason and the tension control mathematical model generation composite machine tension in the BOPP production line, for the constant tension control of composite machine, put forward a kind of improved PID control method based on RBF neural network. By the method of Jacobian information identification of RBF neural network, combined with the incremental PID algorithm to realize the self-tuning tension control parameters, control simulation and implementation of the model using Matlab software programming. The simulation results show that, the improved algorithm has better control effect than the general PID.
\end{abstract}

Keywords: Control PID Algorithm, Jacobian Information Identification, RBF Neural Network, Matlab

\section{Introduction}

Composite machine is a kind of important processing equipment in packaging industry, Non solvent composite has high production efficiency, energy saving, safety, environmental protection and other advantages and gradually attracted the concern of the industry. In which the film high speed, smooth coiling in multi-layer coextrusion drooling film production is especially important. According to these characteristics, this article in order to maintain constant tension as the core, dynamic relationship between the size and speed of tension and linear speed. Study on the variation regularity of receive roll when changing winding motor, by controlling the motor speed difference of indirect tension control. At the same time using RBF neural network PID controller regulates the collection of direct compensation of tension roll speed. So as to realize the high-speed collecting film in roll when changing the constant tension control. Simulation and experiments show that, the presented control method has good control effect.

\subsection{Analysis of Tension and Tension Control}

\subsubsection{Analysis of Composite Machine for Tension}

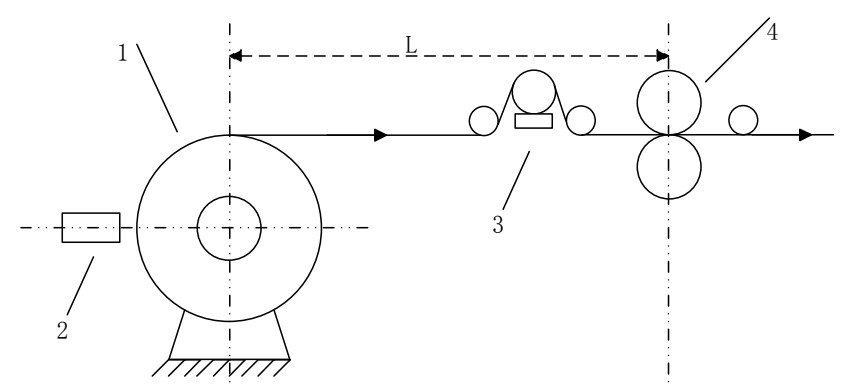

1. Unwinding roller 2. The distance sensor 3. Tension sensor 4.Drag roller

Fig 1 . The reeling tension analysis chart

Tension control system of cast film processing equipment machine usually by the unwinding and rewinding and a series of interstand tension roller, etc.. In order to ensure the wrinkles and cutting the material phenomenon does not occur and to run at a certain speed. The constant tension control play a decisive role. Tension exists mainly in the parent roll unwinding and finished products in the process of winding roll. 
Tension arises mainly from between the film Friction and the reel and the effect of driving force. This paper focuses on the analysis of composite machine control tension, as shown in Figure 1 for the winding tension process analysis.

\subsection{Dynamic Relationship between Tension and Linear Velocity}

Design the raw materials from the unwinding roll out time is $t_{1}$, Line speed is $v_{1}$. The arrival of materials drag roll moments is $\mathrm{t}_{2}$, Line speed is $v$, Elastic modulus of the film is $\mathrm{E}$, the cross-sectional area is $\mathrm{A}$, the hin film from the traction roll to the winding roller length is $\mathrm{L}$, the tension is $\mathrm{F}$. In the period time of $t_{2}-t_{1}$, elongation of the films is $d L=$ $\int_{t_{1}}^{t_{2}}\left(v-v_{1}\right) d t$. According to Hooke's law, film tension can be expressed is:

$$
\mathrm{F}=\mathrm{EA} \frac{\mathrm{dL}}{\mathrm{L}}=\frac{\mathrm{EA}}{L} \int_{\mathrm{t}_{1}}^{\mathrm{t}_{2}}\left(v-v_{1}\right) \mathrm{dt}
$$

By formula (1) shows, we must control the line speed $v-v_{1}$ size to control the film tension of $\mathrm{F}$, Visible tension control system is actually a linear velocity tracking system. Through the control of traction between the roller and the unwinding roller speed differential winding tension to indirect control of film.

\subsection{The Tension Control of Composite Machine}

Tension control system of composite machine is a time-varying, multi input, multi output, nonlinear system. According to the algorithm and improved PID neural network control algorithm of the traditional PID control algorithm, fuzzy control algorithm, the main tension control algorithm composite machine. However, tension control using conventional models are composite machine. If the operating condition changes, the control quality will decline, and even affect the normal operation of the control system.

Kee-hyun Shin proposed an eccentric eccentricity compensation assessment and an adaptive method to eliminate the interference produced by the drum shape ${ }^{[7]}$. Wei Xinming by using BP neural network PID control to reduce the system overshoot and improve the anti-interference performance of the system ${ }^{[8]}$. Chen Zuojie the design of fuzzy PI controller, study the actual value and the tension tension change range. According to the actual value and the change range of size and output of different KP and KI values, and further improve product technology level ${ }^{[9]}$. Deng Xiao uses a single output SPIDNN, enables the system to quickly achieve good dynamic and static performance ${ }^{[10]}$.

Using nonlinear tension control model of the BP neural network has obtained satisfactory results, but because of the multilayer neural network are used in the large amount of computation, slow convergence, and are easy to fall into local minimum point, so the effect of its application. And by radial basis function neural network (RBF) with computational modeling process capability, the algorithm can approximate any nonlinear mapping is simple and practical, small volume, high precision of the model, but also solves the difficult problem of PID parameter setting.

\section{The PID Control of RBF Neural Network}

RBF network is a kind of three layer feedforward neural network for solving pattern classification, including input layer, hidden layer and output layer. The input layer to the hidden layer weights between the input layer is fixed at 1 , which is the information without any treatment ${ }^{[1]}$, only the input variable assigned to the hidden layer, the hidden layer of each neuron represents a radial basis function. The hidden layer to the output layer weights can be adjusted. The hidden layer nodes by like Gauss function radial function as the composition, the number of nodes do not need to like the BP network that are set in advance, but increased in the learning process until it meets the error index so far, the output layer nodes usually is a simple linear function. RBF network PID control consists of two parts: RBF neural network identifier and controller based on PID. Identification RBF neural network through the controlled input and output data to identify the object in an approximate model of the controlled object, and the relationship between input and output instead of the controlled object. The parameters of the PID controller adaptive tuning through identification RBF neural network. RBF network PID control block diagram as shown in figure 2.



Fig 2. Block Diagram of RBF network tuning PID control

\subsection{The Design of the System Structure}

RBF network setting PID control structure as shown in Figure 2, there are two neural network system. Among them, NNC is a self-tuning PID controller, direct close loop control of the controlled object; RBFNNI for online identification system. The realization of controlled Jacobian information recognizing object, on the sensitivity of the control input output control object of observation in order to dynamically, to provide adaptive PID controller. The working principle of the system is based on the controlled object online identification by RBFNNI, power system through the adjustment of NNC in real time, so that the system is adaptive, achieve the purpose of effective control.

\subsection{Design of Neural Network PID Controller}

\subsubsection{Neural Network PID Controller}

PID control is a mature technology, widely used control method, which has the advantages of simple structure, and for most process has better control effect. The discrete PID control law is: 
$\mathrm{u}(\mathrm{k})=K_{P} e(k)+K_{I} \sum_{j=1}^{k-1} e(i)+K_{D}[e(k)-e(k-1)]$

In the formula, $\mathrm{u}(\mathrm{k})$ is the value output controller on $\mathrm{k}$ moment; $\mathrm{K}_{\mathrm{P}}, \mathrm{K}_{\mathrm{I}}, \mathrm{K}_{\mathrm{D}}$ respectively the proportion coefficient, integral coefficient and Differential coefficient; $\mathrm{e}(\mathrm{k})$ as Position and the expectation of AC servo system in current time difference in the value of the moment; $\mathrm{e}(\mathrm{k}-1)$ as Position and expected the last sampling of AC servo system, the difference in the value of the moment.

By formula (3) can be obtained between the increment of control volume $\mathrm{u}(\mathrm{k}-1)$ controller output the first $\mathrm{K}$ cycle time control of the amount of $u(k)$ and the $k-1$ cycle time is:

$$
\begin{gathered}
\Delta \mathrm{u}(\mathrm{k})=\mathrm{u}(\mathrm{k})-\mathrm{u}(\mathrm{k}-1)=K_{P}[e(k)-e(k-1)]+ \\
K_{I} e(k)+K_{D}[e(k)-2 e(k-1)]+e(k-2)
\end{gathered}
$$

The traditional PID control, the main problem is the parameter tuning problem, once the setting calculation of good, are fixed in the whole control process. But in the

practical system, because the system state and parameter changes, the emergence of state and parameters are the uncertainty in the process, the system is difficult to achieve the best control effect. In this paper, using two layers of linear neural network of three parameters of PID controller is adjusted online ${ }^{[2]}$. The input of the neural network is:

$$
\left\{\begin{array}{l}
X_{1}(k)=e(k)-e(k-1) \\
X_{2}(k)=e(k) \\
X_{3}(k)=e(k)-2 e(k-1)+e(k-2)
\end{array}\right.
$$

The performance index was defined as the NNC:

$$
\mathrm{E}(\mathrm{k})=\frac{1}{2}\left[\theta_{d}(k)-\theta(k)\right]^{2}
$$

So:

$$
\begin{gathered}
K_{P}(k)=K_{P}(k-1)-\eta_{1} \frac{\partial E}{\partial K_{P}}=K_{P}(k-1)-\eta_{1}\left[\theta_{d}(k)-\theta(k)\right] \cdot \frac{\partial \theta}{\partial u} \cdot \frac{\partial u}{\partial K_{P}}=K_{P}(k-1)-\eta_{1}\left[\theta_{d}(k)-\theta(k)\right] \frac{\partial \theta}{\partial u} X_{1}(k) \\
K_{I}(k)=K_{I}(k-1)-\eta_{2} \frac{\partial E}{\partial K_{I}}=K_{I}(k-1)-\eta_{2}\left[\theta_{d}(k)-\theta(k)\right] \frac{\partial \theta}{\partial u} X_{2}(k) \\
K_{D}(k)=\mathrm{K}_{D}(k-1)-\eta_{3} \frac{\partial E}{\partial K_{D}}=K_{D}(k-1)-\eta_{3}\left[\theta_{d}(k)-\theta(k)\right] \frac{\partial \theta}{\partial u} X_{3}(k)
\end{gathered}
$$

Among them, $\eta_{1} 、 \eta_{2} 、 \eta_{3}$ is the learning rate, The $\frac{\partial \theta}{\partial u}$ for object Jacobian information, The information can be used by RBFNN network identification.

\subsection{RBF Network Identifier}

In the RBF network identifier structure, $\mathrm{X}=\left[\mathrm{x}_{1}, \mathrm{x}_{2}, \cdots, \mathrm{x}_{n}\right]^{T}$ as the input vectors of the network. Radial basis vector set $\mathrm{H}=\left[\mathrm{h}_{1}, \mathrm{~h}_{2}, \cdots, \mathrm{h}_{j}, \cdots, \mathrm{h}_{m}\right]^{T}$ RBF network for the Gauss function, available:

$$
\mathrm{h}_{j}=\exp \left(\frac{\left\|X-C_{j}\right\|^{2}}{2 b_{j}^{2}}\right)(j=1,2, \cdots, n)
$$

The center vector network of the first $\mathrm{j}$ node $\mathrm{C}_{j}=$ $\left[\mathrm{c}_{j 1}, \mathrm{c}_{j 2}, \cdots, \mathrm{c}_{j i}, \cdots, \mathrm{c}_{j m}\right]^{T} i=1,2, \cdots, n$. Design the base width set for vector network is:

$$
\mathrm{B}=\left[\mathrm{b}_{1}, \mathrm{~b}_{2}, \cdots, \mathrm{b}_{j}, \cdots, \mathrm{b}_{m}\right]^{T}
$$

In the formula: $\mathrm{b}_{j}$ as the width parameter for node $\mathrm{J}$, and a number greater than 0 . The weight vector network is:

$$
\mathrm{W}=\left[\mathrm{w}_{1}, \mathrm{w}_{2}, \cdots, \mathrm{w}_{j}, \cdots, \mathrm{w}_{m}\right]^{T}
$$

The output for the identification network is:

$$
\mathrm{y}_{\text {mout }}(k)=\mathrm{w}_{1} \mathrm{~h}_{1}+\mathrm{w}_{2} \mathrm{~h}_{2}+\cdots+\mathrm{w}_{m} \mathrm{~h}_{m}
$$

In the neural network algorithm composite machine tension control, the control precision demand is not high, not precise part can be modified by adjusting the weight of the network parameters and. The tension of PID controller, the incremental
PID controller, the control error is expressed as:

$$
\operatorname{error}(k)=\mathrm{r}_{\text {in }}(k)-\mathrm{y}_{\text {out }}(k)
$$

Neural network tuning index is:

$$
E(k)=\frac{1}{2} \operatorname{error}(k)^{2}
$$

The control algorithm uses the incremental PID algorithm ${ }^{[3]}$, i.e.:

$$
\begin{array}{r}
\Delta u(k)=\mathrm{k}_{p}[\operatorname{error}(k)-\operatorname{error}(k-1)]+\mathrm{k}_{i}[\operatorname{error}(k)]+ \\
\mathrm{k}_{d}[\operatorname{error}(k)-2 \operatorname{error}(k-1)+\operatorname{error}(k-2)]
\end{array}
$$

Adjustment of $\mathrm{k}_{p} 、 \mathrm{k}_{i} 、 \mathrm{k}_{d}$ by using the gradient descent method, i.e.:

$$
\begin{gathered}
\Delta \mathrm{k}_{p}=-\eta \frac{\partial E}{\partial k_{p}}=-\eta \frac{\partial E}{\partial y_{\text {out }}} \times \frac{\partial y_{\text {out }}}{\partial \Delta u} \times \frac{\partial \Delta u}{\partial k_{p}}= \\
\eta \operatorname{error}(k) \frac{\partial y_{\text {out }}}{\partial \Delta u} x c(1) \\
\mathrm{k}_{i}=-\eta \frac{\partial E}{\partial k_{i}}=-\eta \frac{\partial E}{\partial y_{\text {out }}} \times \frac{\partial y_{\text {out }}}{\partial \Delta u} \times \frac{\partial \Delta u}{\partial k_{i}}= \\
\eta \operatorname{error}(k) \frac{\partial y_{\text {out }}}{\partial \Delta u} x c(2) \\
\mathrm{k}_{d}=-\eta \frac{\partial E}{\partial k_{d}}=-\eta \frac{\partial E}{\partial y_{\text {out }}} \times \frac{\partial y_{\text {out }}}{\partial \Delta u} \times \frac{\partial \Delta u}{\partial k_{d}}= \\
\eta \operatorname{error}(k) \frac{\partial y_{\text {out }}}{\partial \Delta u} x c(1)
\end{gathered}
$$

Type: $\frac{\partial y_{\text {out }}}{\partial \Delta u}$ as the object of the Jacobian information, namely the object output sensitivity to input changes control. The sensitivity is obtained through identification of neural network, i.e.: 


$$
\frac{\partial y_{o u t}}{\partial \Delta u} \approx \frac{\partial y_{\text {mout }}}{\partial \Delta u}=\sum_{j=1}^{m} w_{j} h_{j} \frac{c_{j i}-\Delta u(k)}{b_{j}^{2}}
$$

\section{Tension Control Model and Simulation}

\subsection{The Establishment of tension Control Model}

In the tension of composite machine control, RBF neural network define $\mathrm{n}=3, \mathrm{~m}=6$; Input instruction signals, namely cutting machine winding tension set value $r_{i n}=1$ (Equivalent to the field winding tension of $150 \mathrm{~N})$; Define $\Delta u(k), y_{\text {out }}(k)$ and $y_{\text {out }}(k)-y_{\text {mout }}(k)$ as the input of RBF network identification, among them: $\Delta u(k)$ is PID increment, $y_{\text {out }}(k)$ as the value of cutting machine at the moment the actual output tension. $y_{\text {out }}(k)-y_{\text {mout }}(k)$ Is the difference for cutting machine at the moment the actual output tension and RBF neural network to identify the output tension. To define the network center vector for $\mathrm{C}_{j}=[0,0, \cdots, 0, \cdots, 0]_{n}^{T}$, Base width vector network for $B=[0,0, \cdots, 0, \cdots, 0]_{m}^{T}$, According to the definition of $\eta=3.5$ experience learning rate, the momentum factor $\alpha=0.05$. This paper selects salivation mathematic model of tension control system is:

$$
y_{\text {out }}(k)=\frac{-0.2 y_{\text {out }}(k-1)+u(k-1)}{1+y_{\text {out }}(k-1)^{2}}
$$

To construct the simulation model of the system Using field data to train the RBF neural network controller. Firstly normalized sample data, in the tension control model design. In order to put the 100 groups of measured unreeling constant tension $\quad T=\left[\begin{array}{cccccc}150.00 & 149.90 & 149.80 & 148.90 & 150.00 \cdots \\ 149.90 & 150.00 & 149.90 & 149.80 & 149.90\end{array}\right]$ are mapped to $[0,1]$, select one of the 80 sets of data as training data, and the remaining 20 groups stay on neural network controller test. In this paper, set the data of the minimum value is set to -1 (The number of selected is based on the tension set value is 1 ), Set the data of the middle value is set to 0 . Thus, the measured normalized data can fall in the interval $[0,1]$, get the normalized data after $\mathrm{T}=\left[\begin{array}{lll}1.00 & 0.99 & 0.98\end{array}\right.$

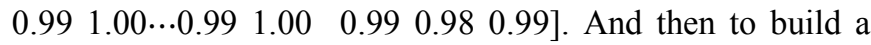
RBF network using radial basis function, which can match with the known sample points. Radial basis function neural network has two layers, that is the hidden layer radial basis neurons and the output layer of linear neurons.

Design radial basis transfer function for the hidden layer of:

$$
\alpha=\operatorname{radbas}(\mathrm{P})
$$

Each neuron in the hidden layer weights and the thresholds of the center position vector of radial basis function $\mathrm{C}_{j}=$ $\left[\mathrm{c}_{j 1}, \mathrm{c}_{j 2}, \cdots, \mathrm{c}_{j i}, \cdots, \mathrm{c}_{j m}\right]^{T}$ and based wide vector $\mathrm{B}=$ $\left[\mathrm{b}_{1}, \mathrm{~b}_{2}, \cdots, \mathrm{b}_{j}, \cdots, \mathrm{b}_{m}\right]^{T}$ corresponding to the specified. Every linear output neurons by weighting these radial basis function and composition. As long as each layer has the correct weights and thresholds, and have enough neurons in hidden layer, function as radial basis function networks to any arbitrary accuracy to approximate arbitrary. The completion of the training of the network in the learning process of neural network set up, in order to achieve the goal of error, the sample point parameter approximation function reaches the target parameter requirements.

\subsection{The Simulation Results of Tension Control}

According to the above are derived by the RBF neural network controller and model identification learning algorithm, using Matlab simulation. The sampling period $\mathrm{t}_{s}=0.001 \mathrm{~s}$. The RBF tuning of PID after the output response as shown in Figure 3, figure $y_{\text {out }}$ for cutting machine at the moment the actual output tension value, and the tension of the given $r_{i n}$ as desired value.

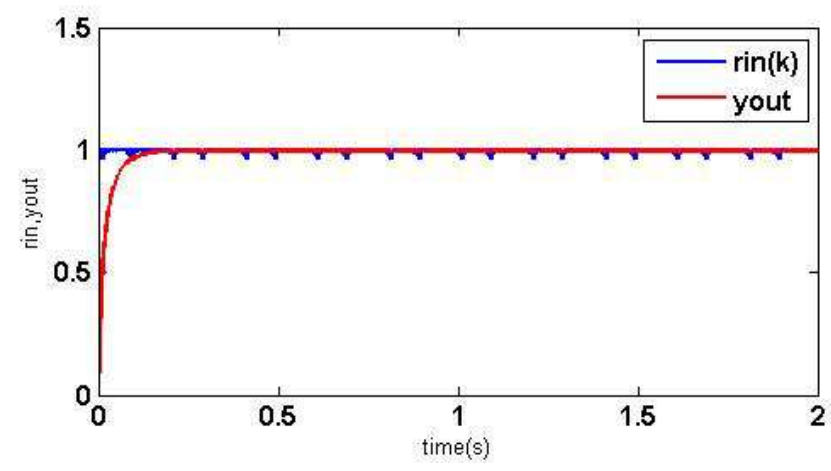

Fig 3. PID output response curves

As you can see from Figure 3, the RBF identification system identification RBF neural network controller has fast response speed, can play fast tracking effect model identification online.

This paper did simulation results were obtained in the absence of any field interference situations, therefore, the stability of the system and this is a scene with no comparable. After the PID tuning output tension curve, the output curve is better in overshoot, adjust time characteristics still than the actual site setup PID more.

As shown in Figure 4 for The traditional PID control curves, these curves reflect the convergence speed is slow. Robustness is not good, we can see that the superiority of RBF neural network PID control.

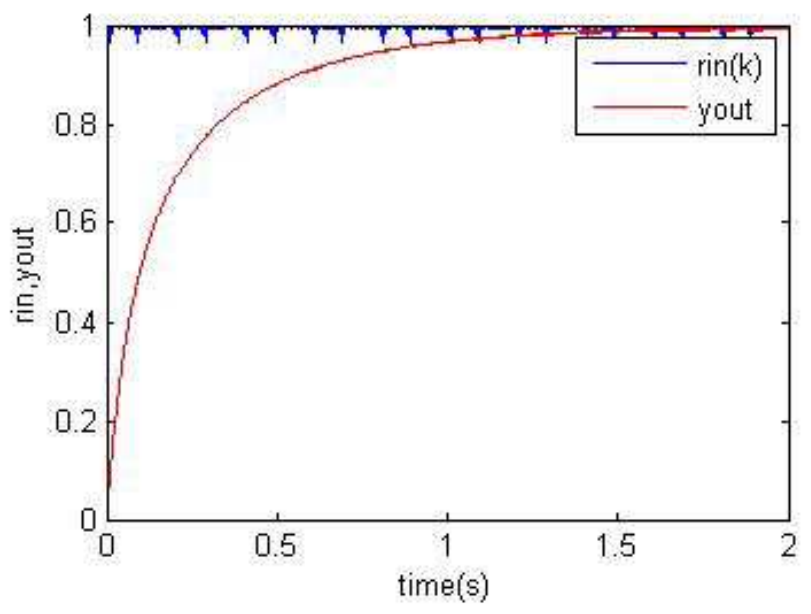

Fig 4. Traditional PID control curves 


\section{Conclusion}

Because the traditional PID control algorithm in the proportion, integral, differential coefficient in the design stage set after week constant, do not have the ability to learn, self adaptive. Therefore, the robustness of using traditional PID tension controller of poor control performance needs to be improved. Simulation results show that PID tension control based on RBF neural network, through online identification process model is established and provide gradient information for the neuron controller, realizes the online identification and online control purposes; and high control accuracy, good dynamic characteristic, has self adaptability and robustness.

\section{References}

[1] Iman Poultangari, Reza Shahnazi ,Mansour Sheikhan RBF neural network based PI pitch controller for a class of 5-MW wind turbines using particle swarm optimization algorithm. ISA Transactions 51 (2012) 641-648.

[2] Ismail Yabanova, Ali Keçebas, Development of ANN model for geothermal district heating system and a novel PID-based control strategy. Applied Thermal Engineering 51 (2013) 908-916.

[3] LIU Hong-mei, WANG Shao-ping, OUYANG Ping-chao, Fault Diagnosis in a Hydraulic Position Servo System Using RBF Neural Network. 2006, 19(4), 346-353.
[4] Shi Zhongzhao. Neural network control theory [M]. Xi'an: Northwestern Polytechnical University press, 1999:85-90.

[5] Wang Jiangjiang, Zhang Chunfa, Jing Youyin. Self-adaptive RBF neural network PID control in exhaust temperature of micro-turbine[C] Proceedings of the Seventh International Conference on Machine Learning and Cybernetics,2008:2131-2136.

[6] Elanayar V T S, Shin Y C. Radial basis function neural network for approximation and estimation of nonlinear stochastic dynamic systems [J].IEEE Transactions on Neural Network, 1994, 5 (4):594-603.

[7] Kee-Hyun Shin, Jeung-In Jang, et al. Compensation Method for Tension Disturbance Due to an Unknown Roll Shape in a Web Transport System [C].IEEE Transactions on industry applications. 2003, 5(39): 1422-1428.

[8] Wei Xinming. Take the tension control system for machine based on BP neural network volume [D]. Northeastern University. 2014

[9] Deng Xiao, Hu Muyi, PID neural network application in the roll paper roll tension control system. China paper journal [J]. 2014.29(1).44-48

[10] Chen Zuojie, Wu Peide, Zhang Yihong. Application of fuzzy PI controller in the study of film tension control system [J]. 2014.3(1).42-44 